\title{
Vaginal transmission of cell-associated HIV-1 in the mouse is blocked by a topical, membrane-modifying agent
}

\author{
Kristen V. Khanna, ${ }^{1,2}$ Kevin J. Whaley, ${ }^{2,3,4}$ Larry Zeitlin, ${ }^{4}$ Thomas R. Moench, ${ }^{2}$ \\ Karim Mehrazar, ${ }^{5}$ Richard A. Cone,,${ }^{2,3}$ Zhaohao Liao, ${ }^{6}$ James E.K. Hildreth, ${ }^{6}$ \\ Timothy E. Hoen, ${ }^{3}$ Leonard Shultz, ${ }^{7}$ and Richard B. Markham ${ }^{1}$ \\ ${ }^{1}$ Johns Hopkins School of Hygiene and Public Health, Department of Microbiology and Immunology, \\ Johns Hopkins University, Baltimore, Maryland, USA \\ ${ }^{2}$ ReProtect LLC, Baltimore, Maryland, USA \\ ${ }_{3}^{3}$ Johns Hopkins University, Department of Biophysics, Baltimore, Maryland, USA \\ ${ }^{4}$ Epicyte Pharmaceuticals Inc., San Diego, California, USA \\ ${ }^{5}$ Morgan State University, Medical Technology Program, Department of Biology, Baltimore, Maryland, USA \\ ${ }^{6}$ Johns Hopkins School of Medicine, Department of Pharmacology and Molecular Sciences, Baltimore, Maryland, USA \\ ${ }^{7}$ The Jackson Laboratory Inc., Bar Harbor, Maine, USA \\ Address correspondence to: Kristen V. Khanna, Johns Hopkins School of Hygiene and Public Health, \\ Department of Molecular Microbiology and Immunology, 615 North Wolfe Street, \\ Room E-5132, Baltimore, Maryland 21205, USA. \\ Phone: (410) 955-9280; Fax: (410) 614-8263; E-mail: kkhanna@jhsph.edu.
}

This work was presented, in part, at the Twelfth World AIDS Conference in Geneva, Switzerland, June 28-July 3, 1998.

Received for publication May 10, 2001, and accepted in revised form November 26, 2001.

Because both HIV-1 virions and HIV-infected cells are present in the semen and cervical mucus of infected individuals, HIV-1 prevention strategies must consider both cell-free and cell-associated virus. Antibodies that target HIV-1 virions have been shown to prevent vaginal transmission of cellfree virus in macaques, but since cell-associated transmission has not been reliably demonstrated in this model system, no strategies to prevent such transmission have been tested. We have employed a mouse model in which SCID mice carry human peripheral blood leukocytes (HuPBLs). In these mice, vaginal transmission of cell-associated, but not cell-free, HIV-1 transmission occurs, mediated by transepithelial migration of HIV-infected cells. Topical application of $\beta$-cyclodextrin ( $\beta$-CD), a cholesterol-sequestering agent that interferes with cell migration and budding of virus from lipid rafts, blocks transmission of cell-associated HIV-1. The HuPBL-SCID model of vaginal HIV-1 transmission should prove useful for investigating cell-associated HIV-1 transmucosal HIV-1 transmission, as well as for screening reagents for their potential efficacy in preventing sexual HIV-1 transmission.

J. Clin. Invest. 109:205-211 (2002). DOI:10.1172/JCI200213236.

\section{Introduction}

Newly acquired HIV-1 infections are largely the result of heterosexual contact (1). While mechanical barriers such as condoms can be effective in preventing sexual transmission of HIV-1, this method is not always accepted by male partners or is otherwise impractical for use by women. The initial evaluation of alternative prevention strategies, particularly those using techniques that can be controlled by women, requires the use of model systems of vaginal HIV-1 transmission.

In this report we describe a model of vaginal transmission in human peripheral blood leukocyte-reconstituted (HuPBL-reconstituted), severe combined immunodeficient (SCID) mice. This model should prove useful because it (a) provides the capacity to screen large numbers of animals to determine the statistical robustness of any observations made; (b) uses the pathogen of interest, HIV-1; and (c) mimics a critical feature of clinical transmission (i.e., preferential transmission of viruses that use chemokine receptor-5 [CCR5] as a coreceptor). Impor- tantly, this model specifically addresses the role of cellassociated transmission of HIV-1. Cell-associated transmission by the vaginal route has been difficult to achieve and remains controversial in nonhuman primate models using simian immunodeficiency virus (SIV) $(2,3)$. Because semen from HIV-1-infected men (4) and cervical mucus from HIV-1-infected women (5) contain both cell-free virus and HIV-infected cells, both may be important for sexual transmission of HIV-1. Furthermore, in vitro model systems have consistently shown that transmission of cell-associated virus is more efficient than that of cell-free virus. Thus, models for studying the transmission of cell-associated virus, which would not be expected to be susceptible to virus-specific intervention strategies, are critically needed.

Interruption of cell-associated transmission requires an understanding of the processes by which virus moves from infected donor cells to cells of the recipient. The migration of HIV-infected cells and the movement of assembled virus particles out of the infected donor cell are 
critical to this process. Recent studies have indicated that budding of HIV-1 occurs selectively through lipid rafts on the cell surface (6). In addition, the ability of lipid rafts to act as adhesion platforms has been shown to facilitate cellcell interactions (7) and migration (8), which may be important for cell-to-cell transfer of virus and for entry of infected cells through genital tract epithelia, respectively.

$\beta$-Cyclodextrins are water-soluble compounds that disrupt lipid rafts by adsorbing cholesterol from cellular membranes $(9,10)$. They have been shown to interrupt cellular migration by blocking the establishment of cellular polarity that is required for migration to occur (11, 12). The current study uses the HuPBL-SCID mouse model to examine the ability of 2-hydroxypropyl $\beta$-cyclodextrin (2OHp- $\beta$-CD) to interrupt cell-associated transmission of HIV-1. Our results indicate that $2 \mathrm{OHp}-\beta$-CD administered intravaginally prior to infected-cell challenge efficiently blocks virus transmission and induces minimal, if any, damage to the vaginal mucosa.

\section{Methods}

Cell culture. Blood was obtained from HIV-negative volunteers at the Johns Hopkins University Hemapheresis Laboratory. HuPBMCs were isolated by centrifugation on Ficoll-Hypaque gradient (Pharmacia Biotech AB, Uppsala, Sweden) and were washed and suspended at $5 \times 10^{7} / \mathrm{ml}$ in PBS prior to intraperitoneal administration to SCID mice. HuPBMCs that were used as inocula were maintained in RPMI-1640 (Life Technologies Inc., Grand Island, New York, USA), supplemented with 10\% FCS, penicillin-streptomycin, and gentimicin (all supplements from Life Technologies Inc.), hereafter referred to as complete RPMI (cRPMI). PBMCs were stimulated with phytohemagglutinin (PHA; Sigma Chemical Co., St. Louis, Missouri, USA) for 2 days; cells were then exposed to 300 fifty-percent tissue culture infectious doses $\left(\mathrm{TCID}_{50}\right)$ of HIV- $1_{\mathrm{Ba}-\mathrm{L}}$ in cRPMI with IL-2 (10 $\mathrm{U} / \mathrm{ml}$; Boehringer Mannheim Biochemicals Inc., Indianapolis, Indiana, USA). Infected-cell cultures were maintained in cRPMI supplemented with IL-2 for 10 days prior to inoculation into the mice. Limiting dilution-PCR (LD-PCR) was performed using HIV-1 gagspecific primers as described previously (13) to determine the extent to which HuPBMCs were infected with HIV-1. To assess virus recovery from cells harvested from the peritoneal cavities of challenged mice, uninfected HuPBMCs were stimulated with PHA and maintained in IL-2-supplemented media $\left(1 \times 10^{6}\right.$ per mouse $)$ in preparation for coculture with the peritoneal cells recovered from the HuPBL-SCID mice.

HIV-1 virus preparation. A single lot of the inoculum virus,

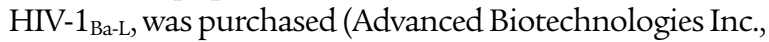
Columbia, Maryland, USA). Virus was aliquoted and stored in liquid $\mathrm{N}_{2}$ until used to infect HuPBMCs. The $\mathrm{TCID}_{50}$ inoculated was confirmed using the MAGI assay (MAGI-CCR5, a HeLa-CD4 cell line that expresses CCR5 and that has an integrated copy of the HIV-1 long terminal repeat-driven $\beta$-D-galactosidase reporter gene), and by titration on peripheral blood-derived monocytes.
Vaginal infection of HuPBL-SCID mice with HIV-1. Female mice with severe combined immunodeficiency (C.B-17 SCID) $(14,15)$ were obtained from Charles River Laboratories (Wilmington, Massachusetts, USA) or from our SCID mouse colony (established using C.B-17 mice from The Jackson Laboratory Inc.). The mice were treated subcutaneously with $2.5 \mathrm{mg}$ progestin (Depo-Provera; Pharmacia Corp., Peapack, New Jersey, USA) on the same day as administration of $5 \times 10^{7}$ unstimulated HuPBMCs intraperitoneally in $1 \mathrm{ml}$ PBS. Seven days following progestin treatment and reconstitution of the SCID mice with HuPBMCs, the mice were anesthetized and administered pelleted, cell-free HIV-1 $1_{\mathrm{Ba}-\mathrm{L}}$ (up to $10^{6} \mathrm{TCID}_{50}$ ), or supernatant fluids from HIV- $1_{\mathrm{Ba}-\mathrm{L}}$-infected HuPBLs, HIV- $1_{\text {Ba- }}$-infected HuPBLs, or HIV- $1_{\mathrm{MN}}$-infected HuPBLs $\left(1 \times 10^{6}\right.$ per mouse). In all cases in groups receiving HIVinfected PBLs as inoculum, the cells were obtained from a donor other than that from which cells were obtained for the original transplant into the peritoneal cavities of the mice. In the $\beta$-CD experiments, the mice received either $\beta$-CD (3\% wt $/ \mathrm{vol}$ in PBS) 5 minutes prior to receiving $1 \times 10^{6}$ HIV-1 $1_{\mathrm{Ba}-\mathrm{L}}$-infected HuPBLs; $1 \times 10^{6}$ HIV- $1_{\mathrm{Ba}-\mathrm{L}}$-infected HuPBLs preincubated with $3 \% \beta-\mathrm{CD}$; or $1 \times 10^{6} \mathrm{HIV}-1_{\mathrm{Ba}-\mathrm{L}}$-infected HuPBLs suspended in PBS. Mice remained anesthetized for 5 minutes following intravaginal inoculation by pipette. Extreme care was taken to avoid causing trauma to vaginal tissues. Two weeks later the mice were euthanized and peritoneal cells were recovered by lavage with cold PBS. The cells recovered by lavage (of both murine and human origin) were assayed by DNA-PCR for human $\beta$-globin to confirm the success of the human cell engraftment in the mice and to assay for the presence of HIV-1 in the recovered HuPBMCs, which was also determined by coculture of recovered peritoneal cells with uninfected, PHA-stimulated HuPBMCs.

Vaginal epithelial morphology. Four BALB/c and four HuPBL-SCID mice were sham-treated or treated with $2.5 \mathrm{mg}$ Depo-Provera 1 week prior to the experiment. Mice were euthanized and reproductive tissues were collected and dissected. Excised vaginal tissue was fixed (Omnifix; Zymed Laboratories Inc., South San Francisco, California, USA) overnight and embedded in paraffin, sectioned, and stained with hematoxylin and eosin.

Fluorescent in situ bybridization. Six SCID mice were treated with $2.5 \mathrm{mg}$ progestin, with or without HuPBL reconstitution (i.e., peritoneal transplant of human cells). Spleen with peritoneal mesentery, and vaginal tissues, were fixed in paraformaldehyde and embedded in paraffin. Sections were mounted on slides, deparaffinized, and made permeable by immersion in $50 \%$ glycerol in $0.1 \times \mathrm{SSC}$ at $90^{\circ} \mathrm{C}$, followed by incubation in protease solution (16). Sections were then co-denatured with a biotin-labeled, human pan-centromere probe (Cytocell Ltd., Banbury, United Kingdom) at $75^{\circ} \mathrm{C}$, and hybridized overnight. Slides were then washed, and bound probe was detected with CY3-conjugated streptavidin (Cytocell). Tissue sections were counterstained with DAPI, and examined and photographed with epifluorescence microscopy. 


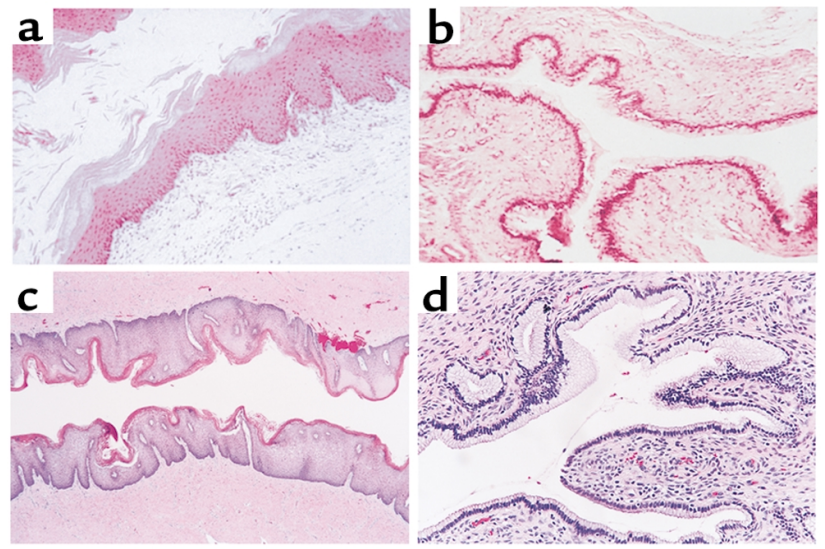

Figure 1

Progesterone treatment of C.B-17 SCID mice thins the vaginal epithelium. Female C.B-17 scid/scid mice, or BALB/c mice (not shown) were given either saline or $2.5 \mathrm{mg}$ Depo-Provera and euthanized after 7 days. Tissues were collected and processed as described in Methods. Tissue sections shown are hematoxylin and eosin-stained vaginal epithelium from (a) an untreated mouse in estrus $(\times 120)$, (b) a progestin-treated mouse $(\times 120),(\mathbf{c})$ normal human vagina $(\times 50)$, and $(\mathbf{d})$ normal human cervix $(\times 160)$. While there are striking differences between the progestin-treated vaginal epithelium of a SCID mouse and the untreated, stratified, squamous vaginal epithelium (murine), the similarities in morphology and cellular architecture are apparent between the thin, columnar, progestin-treated, murine vaginal epithelium and the human, columnar, cervical epithelium.

Vaginal epithelial toxicity. CF-1 mice were pretreated with $2.5 \mathrm{mg}$ progestin; 1 week later, three groups of three mice each were inoculated with $50 \mu$ l of either PBS, $1 \%$ (wt/vol) nonoxynol-9, or 3\% (wt/vol) $\beta$-CD ( 20 mM; Cyclodextrin Technologies Development Inc., Gainesville, Florida, USA). Each test solution also contained the membraneimpermeant DNA-binding fluorescent dye ethidium bromide homodimer-1 (20 $\mathrm{MM}$; Molecular Probes Inc., Eugene, Oregon, USA) (17). Fifteen minutes after exposure to the test agents, the mice were euthanized, and the vaginas were dissected, opened longitudinally, and viewed by fluorescent microscopy using a TRITC filter set.

\section{Results}

Use and effect of progesterone pretreatment in establishing a murine model of vaginal HIV-1 transmission. Studies in nonhuman primate models of SIV or chimeric simianhuman immunodeficiency virus (SHIV) transmission have frequently pretreated the challenged animals with progesterone $(2,18,19)$, with the stated purpose of synchronizing the estrous cycle among experimental animals. This treatment also alters the cellular architecture of the vaginal epithelium, facilitating viral transmission by this route $(2,19)$. Progestin treatment of HuPBL-SCID mice similarly caused the multilayer, stratified, squamous epithelium of the untreated mouse vagina (Figure 1a) to assume a cervixlike, singlelayer, columnar morphology (Figure 1b). Thus progestin treatment has the effect of rendering the mouse vagina morphologically unlike the human vagina (Figure 1c) and similar to human, columnar cervical epithe- lium (Figure 1d), which in organ culture is more readily infected with HIV-1 than is vaginal tissue (20). In a series of pilot studies (data not shown), it was determined that neither cell-free nor cell-associated HIV-1 could be transmitted in HuPBL-SCID mice by the vaginal route without prior administration of progestin (medroxyprogesterone acetate, Depo-Provera).

$H u P B L-S C I D$ mice are susceptible to vaginal transmission of cell-associated, but not cell-free, HIV-1. To determine whether HuPBL-SCID mice, transplanted intraperitoneally with $5 \times 10^{7}$ uninfected, unstimulated human PBMCs 1 week earlier, were susceptible to cell-associated or cell-free virus, mice were exposed vaginally to cell-free $\mathrm{HIV}-1_{\mathrm{Ba}-\mathrm{L}}$ (CCR5-utilizing strain) and

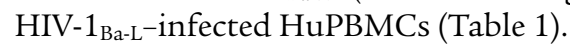

High-titer, cell-free HIV-1 and virus obtained from supernatant fluid from the HIV-1-infected HuPBMCs used in the same experiment (i.e., recently budded virus) were tested. HIV-1-infected HuPBMCs were prepared for inoculation into the mice 10 days after in vitro exposure of PHA- and IL-2-stimulated cells to HIV-1. Despite intravaginal inoculation of up to $1 \times 10^{6.5}$ TCID $_{50}$ of cell-free HIV-1 $1_{\text {Ba-L }}$, virus was not transmitted to the HuPBMCs that had been transplanted intraperitoneally in the mice. However, cell-associated HIV- $1_{\mathrm{Ba}-\mathrm{L}}$ was efficiently transmitted vaginally in the HuPBLSCID mice with as few as 250,000 HuPBMCs, between $1 \%$ and $5 \%$ of which were infected with HIV-1 (i.e., as few as $10^{3.5} \mathrm{HIV}-1$-infected cells). HuPBMCs infected to similar levels with $\mathrm{HIV}_{\mathrm{MN}}$, a CXCR4-using variant, and inoculated intravaginally transmitted infection much less efficiently (Table 2).

Vaginal inoculation of HIV-1-infected PBMCs does not induce visible damage to the vaginal epithelium. While great care was taken to avoid causing damage to the reproductive epithelium upon administration of HIV-1-infected PBMCs, histology was performed on the vaginal epithelium to demonstrate the absence of gross cellular damage (Figure 2) following administration of tissue culture medium containing an irrelevant protein.

\section{Table 1}

Cell-associated virus is transmitted in the HuPBL-SCID mouse model of vaginal HIV-1 transmission

HIV inoculum $\quad \begin{gathered}\text { Number of mice from which HIV-1 was cultured/ } \\ \text { Number of mice exposed to HIV-1 }\end{gathered}$

HIV-1 $1_{\text {Ba-L-infected HuPBMCs }}$

$1.00 \times 10^{6}$ cells

$0.25 \times 10^{6}$ cells

$0.10 \times 10^{6}$ cells

$0.05 \times 10^{6}$ cells

$0.01 \times 10^{6}$ cells

$56 / 66^{A}$
$4 / 5^{A}$
$4 / 9$
$1 / 5$
$0 / 9$

HIV-1 $1_{\text {Ba-L }}$ cell-free virus

$1.0 \times 10^{6.5} \mathrm{TCID}_{50} \quad 0 / 7$

$1.0 \times 10^{6} \mathrm{TCID}_{50} \quad 0 / 5$

$1.0 \times 10^{5} \mathrm{TCID}_{50} \quad 0 / 5$

AP $<0.05$ compared with cell-free virus. 
Table 2

CCR5-utilizing strains of HIV-1 are transmitted vaginally in HuPBL-SCID mice $^{\mathrm{A}}$

HIV inoculum Number of mice from which HIV-1 was cultured/ Number of mice exposed to HIV-1

HIV-1 $1_{\text {Ba-L }}($ R5 $)$-infected HuPBMCs

$1.00 \times 10^{6}$ cells $\quad 5 / 5^{\mathrm{B}}$

$0.25 \times 10^{6}$ cells $\quad 4 / 5^{\mathrm{B}}$

$0.05 \times 10^{6}$ cells $\quad 1 / 5$

HIV- $1_{\text {MN }}($ X4)-infected HuPBMCs

$1.00 \times 10^{6}$ cells $\quad 1 / 5$

$0.25 \times 10^{6}$ cells $\quad 0 / 5$

$0.05 \times 10^{6}$ cells $\quad 0 / 5$

ARepresentative of two separate experiments with five mice per group. ${ }^{\mathrm{B}} P<0.05$ compared with an equal number of HIV- $1_{\mathrm{MN}}$-infected HuPBLs.

Human PBMCs transplanted into the HuPBL-SCID peritoneal cavity do not populate the reproductive tract. To determine whether human cells placed into the peritoneal cavity could migrate to sites in the vaginal mucosa and/or submucosa, HuPBMCs were transplanted into the peritoneal cavities of progesterone-treated female HuPBL-SCID mice. Seven days later the mice were euthanized and tissue sections of the vagina, spleen, and peritoneal mesentery were hybridized with a human pan-centromere probe to detect human cells. Whereas abundant human cells were found in the peritoneal mesentery (Figure 3a), and occasionally in the spleen of all the HuPBL-SCID mice (not shown), no human cells were detected in the vaginal tissues (Figure 3b). Thus, in this model, there appear to be no locally accessible target cells in the vagina that free virus could infect following intravaginal inoculation.

Human PBMCs introduced vaginally migrate to the regional lymph nodes of HuPBL-SCID mice. To define the basis for transmission of cell-associated virus, we examined whether HuPBMCs, both uninfected and HIV-1-infected, were capable of migrating from the vagina to the site of transplanted human cells in the peritoneal cavity. HuPBMCs were labeled with bisbenzamide $(3 \mu \mathrm{g} / \mathrm{ml})$; the fluorescent human cells $\left(1 \times 10^{7}\right)$ were added vaginally to Depo-Provera-treated mice, and 4 hours later the iliac lymph nodes of each mouse were removed and homogenized on a cell strainer. Fluorescent cells were detected in the lymph nodes (mean cell number 204, ranging from 0 to 395 , up to $5 \%$ of the cells recovered from the lymph nodes of SCID mice). The human cells migrated to the iliac lymph nodes of both progestin-treated BALB $/ \mathrm{c}$ and progestin-treated HuPBL-SCID mice (data not shown).

$\beta$-CD prevents cell-associated HIV-1 transmission and is not toxic to the vaginal epithelium. This model could be used to address specifically the problem of inhibiting cell-associated transmission. Since $\beta$-CD has been shown to inhibit cell migration $(11,12)$ as well as inhibit the infectivity of HIV-1-infected cells (21), we tested the ability of $\beta$-CD to inhibit vaginal transmission of cell-associated HIV-1. Reconstituted HuPBL-SCID mice were challenged intravaginally with $1 \times 10^{6} \mathrm{PBMCs}$ infected with
$\mathrm{HIV}_{\mathrm{Ba}-\mathrm{L}}$ after receiving progesterone subcutaneously and HuPBMCs intraperitoneally. For two of the experimental groups the HIV-1-infected PBMCs were preincubated in $20 \mu \mathrm{l}$ of either PBS or $2 \mathrm{OHp}-\beta-\mathrm{CD}(3 \% \mathrm{wt} / \mathrm{vol})$ before the mixture was inoculated intravaginally. A third group of mice were given $20 \mu \mathrm{l} 2 \mathrm{OHp}-\beta-\mathrm{CD}(3 \%)$ intravaginally followed 5 minutes later by infected PBMCs in PBS $(10 \mu \mathrm{l})$. The results (Table 3) indicate that $\beta$-CD significantly inhibited cell-associated HIV-1 transmission by this route, even when administered prior to exposure to HIV-1-infected PBMCs.

To examine the effect of $\beta$-CD on the vaginal epithelium, CF-1 mice were pretreated with progesterone and 1 week later were inoculated with $50 \mu \mathrm{l}$ of PBS, $1 \%$ (wt/vol) nonoxynol-9, or $3 \%(\mathrm{wt} / \mathrm{vol}) \beta-\mathrm{CD}(\sim 20 \mathrm{mM})$ containing the membrane-impermeant DNA-binding fluorescent dye ethidium bromide homodimer-1. The vaginas of the mice were viewed by fluorescent microscopy. While 1\% nonoxynol-9 (Figure 4a) caused considerable epithelial damage as indicated by the membrane-damaged cells that are fluorescent, the vaginal epithelial cells of $\beta$-CD-treated mice had little membrane damage (Figure $4 \mathrm{~b}$ ) and appear more similar to the vaginal epithelium of the negative control, PBS-treated mice (Figure 4c).

\section{Discussion}

Several mechanisms have been proposed by which HIV-1 is able to traverse the epithelium of the genitourinary tract to establish productive infection in lymph nodes. Studies have suggested that HIV-1 can be transmitted from infected lymphocytes to epithelial cells $(22,23)$, or through the epithelium, which serves as a conduit through which virus is transcytosed, presumably to cells within the lamina propria that are susceptible to productive infection (24). In vivo intravaginal inoculation of rhesus macaques with SIV has demonstrated rapid association of the virus with dendritic cells adjacent to or between the epithelial cells lining the genitourinary tract $(25,26)$, or with quiescent
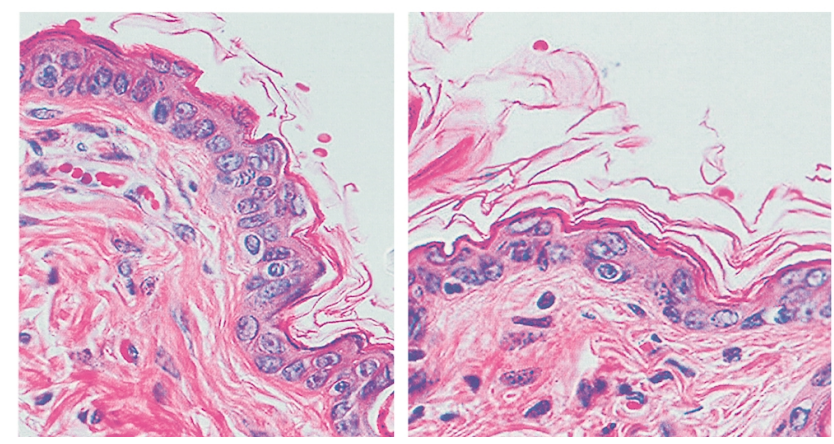

\section{Figure 2}

SCID mice treated with progestin have intact vaginal epithelium following administration of medium containing an irrelevant protein with a pipette. Histology was performed as described in Methods. Tissue sections were fixed overnight and embedded in paraffin, sectioned, and stained with hematoxylin and eosin. 

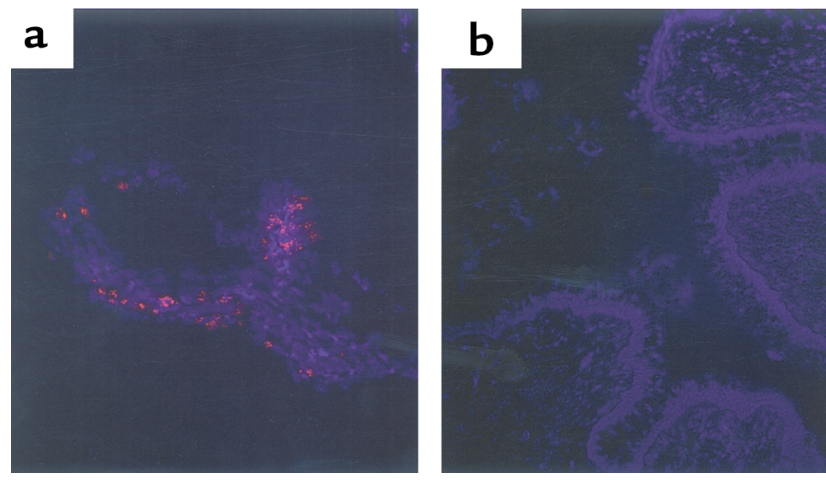

\section{Figure 3}

Human cells do not populate the HuPBL-SCID mouse vagina. HuPBMCs were transplanted into the peritoneal cavity of progesterone-treated, female HuPBL-SCID mice. Tissue sections of the vagina, spleen, and peritoneal mesentery taken 7 days later were hybridized with a human pan-centromere probe to detect human cells. Whereas abundant human cells were found in the peritoneal mesentery (a), and occasionally in the spleen (not shown), no human cells were detected in the vaginal tissues $(\mathbf{b})$. No human cells were detected in an additional negative control, a mouse that did not receive a HuPBL transplant (not shown).

$\mathrm{T}$ cells similarly positioned in the reproductive tract (27). All of these mechanisms of transmission involve some exposure of free virus to the extracellular environment, providing an opportunity, albeit possibly quite brief (26), for virus-specific intervention strategies to be effective at the mucosal surface.

Of additional concern, however, is the possibility that lymphocytes or macrophages from the infected donor could migrate directly through the epithelium of the genitourinary tract to infect lymphocytes in lymph nodes draining the genitourinary tract. We argue that anti-HIV antibodies or other virion-specific strategies, while important and perhaps necessary for a protective effect, may not be sufficient.

Previous references to such migrating cells have referred to them as "Trojan horse" leukocytes because of their ability to hide virus from virus-specific defenses that might exist within the genitourinary tract (28). While considerable effort has been directed toward the identification of virus-specific intervention strategies that would be effective against sexual transmission of human and simian immunodeficiency viruses $(18,29,30)$, there has been little effort to identify strategies for interrupting migration of infected cells to regional lymph nodes.

The mouse model of vaginal transmission presented here demonstrates the vaginal transmission of HIV-1 using infectedcell inocula. The HuPBL-SCID model is unique in that the processes of cell-associated HIV-1 transmission can be examined in the absence of the possibility that cellfree virus is mediating transmission. In fact, the amount of infectious virus produced by the number of infected cells in our inocula would be predicted (31) to be dramatically less than the $1 \times 10^{6} \mathrm{TCID}_{50}$ of free virus that failed to infect the mice.

Rather, in this model, HIV-1-infected PBMCs are able to migrate through cervixlike epithelium to regional lymph nodes. Therefore this system can be used to evaluate strategies that may be effective in blocking cell-associated transmission. To date, cell-associated SIV has not been successfully transmitted by the vaginal route in a macaque model (2), although both cell-free and cellassociated HIV-1 have been transmitted by viral inoculations at the cervical os of chimpanzees (3). Similarly, both cell-free and cell-associated feline immunodeficiency virus have been transmitted in cat models of vaginal infection $(32,33)$.

In the current model, only CCR5-utilizing HIV-1 could be efficiently transmitted and establish infection in the HuPBMCs that had been previously transplanted intraperitoneally into the mice. It is unclear whether this preferential transmission reflects preferential movement of CCR5-utilizing virus-infected cells across the mucosal barrier or an enhanced ability of these viruses to continue productive infection in the unactivated HuPBMCs residing in the peritoneal cavity 7 days after human cell transplantation (34). This finding does, however, parallel the observation that viruses that can use CCR5 as a coreceptor for entry are preferentially transmitted in the clinical setting.

Unlike other model systems of vaginal transmission, the HuPBL-SCID mouse model of transmission is dependent upon the movement of virus-infected cells to sites at which other human cells exist, in this case the peritoneal cavity of the infected mice. We have not examined whether transmission or persistent virus infection is observed in the absence of human target cells in the peritoneal cavity. In the clinical setting, allogeneic target cells would be present in the lymph nodes to which these cells have been observed to migrate.

Human cells transplanted into the peritoneum do not appear to migrate to the vaginal mucosa or submucosa. Thus the failure of free virus to be transmitted in this system may simply reflect the poor migratory ability of free virus and the absence of human target cells within and beneath the vaginal mucosa. These findings, therefore, do not indicate that free virus is not transmitted in the clinical setting, but rather demonstrate that infected-cell migration through cervical epithelium must be considered in any intervention strategy.

\section{Table 3}

Ability of $\beta-C D$ to inhibit vaginal transmission of cell-associated HIV-1 in HuPBLSCID mice

Treatment of HIV-infected cells Number of mice from which HIV-1 was cultured/ Number of mice exposed to HIV-1

HuPBMCs premixed with PBS HuPBMCs premixed with $\beta-C D$ $\beta-C D$ administered intravaginally prior to infected HuPBMC challenge

AP $<0.01$ compared with HuPBMCs premixed with PBS. 

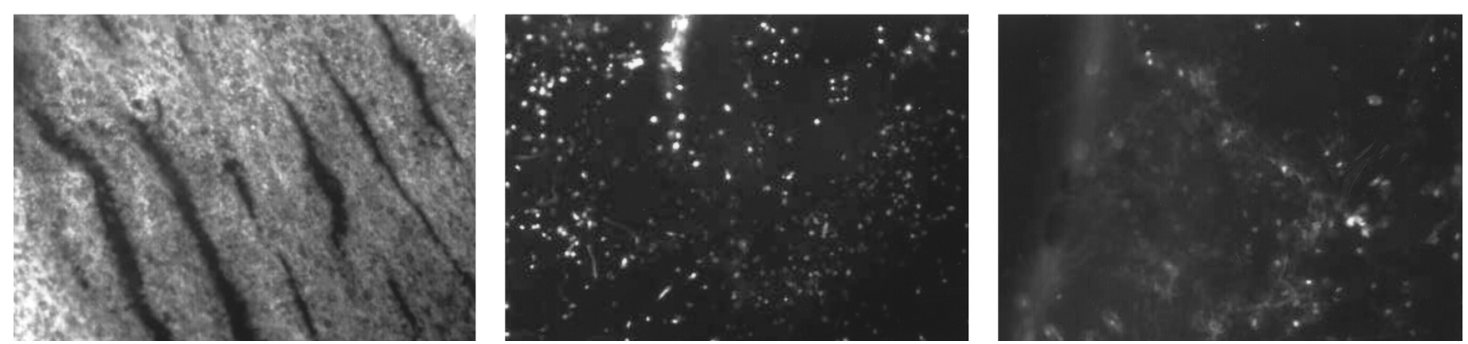

Figure 4

$\beta-C D$ is not toxic to murine vaginal epithelium. Depo-Provera-treated, CF-1 mice were treated vaginally with $50 \mu$ l of (a) $1 \%$ nonoxynol-9 (positive control), (b) $3 \% \beta-\mathrm{CD}$, or (c) PBS (negative control). The membrane-impermeant dye ethidium bromide homodimer-1 (20 $\mu \mathrm{M}$ ) was included to determine cellular viability after 15 minutes' exposure to the test agents. The vaginas were dissected and opened longitudinally and were viewed and photographed by fluorescence microscopy using a TRITC filter set.

The migration of mononuclear cells through murine vaginal epithelium has been previously documented (35-37). The current studies reinforce the notion that the single layer of columnar epithelial cells present on the surface of the cervix is more susceptible to transmigration of HIV-infected PBMCs and, conversely, that the stratified squamous epithelium lining the normal vagina is less vulnerable to transepithelial transmission, presumably by reducing the efficacy of transepithelial migration. Progesterone treatment of the mice effectively converts the vaginal stratified squamous epithelium into a cervixlike columnar epithelium, thus greatly increasing the surface area within the murine vagina that is covered with columnar epithelium. In the clinical setting a much larger human cervical epithelial surface would be available for such transmission.

Using the HuPBL-SCID model of vaginal transmission, we have identified a candidate agent that is highly effective in interrupting vaginal transmission of cell-associated HIV-1. Application of $\beta$-CD to the vaginal mucosa prior to inoculation with HIV-1-infected cells dramatically reduced transmission of cell-associated virus. $\beta$-Cyclodextrins are cyclic, water-soluble carbohydrates composed of seven glucose units (38) and have been used clinically as food additives (38), and as molecular complexing agents that can increase the solubility and stability of some poorly soluble drugs, which have then been administered by the intravenous route (39). As might be expected from the systemic tolerance of $\beta$-cyclodextrins in the human clinical setting, the current studies indicate that $2 \mathrm{OHp}-\beta-\mathrm{CD}$ applied to the vaginal mucosa was substantially less toxic than a subclinical concentration of the widely used spermicide nonoxynol-9.

It is likely that migration through the epithelium involves, as an initial step, interaction between lymphocytes and/or macrophages and epithelial cells. Clustering of lipid rafts on the cell membrane results in enhancement of cell binding and migration (8), and conversely, disruption of those rafts with $\beta$-CD diminishes cell-cell interactions and migration $(11,12)$. Moreover, the production of HIV-1 virions from such cholesterol-depleted cells is dramatically decreased, and these virions are significantly less infectious (21). Alternatively, or in addition, $\beta$-CD has been shown to dis- rupt signaling events and pathways that may be critical for the process of transepithelial transmission of HIV-1. Relevant to the current studies are the observations that disruption of lipid rafts prevents cells from developing the polarity required for cell migration (8).

The current studies indicate that the mouse model of vaginal transmission of cell-associated virus provides a simple and inexpensive model to identify agents that may be necessary components of vaginal products for preventing the sexual transmission of HIV. These studies demonstrate that $2 \mathrm{OHp}-\beta$-CD significantly blocks vaginal transmission of cell-associated HIV-1 and warrants further study in the preclinical and clinical settings.

\section{Acknowledgments}

The authors thank Gene Barbour and David Ford for outstanding technical assistance, the animal care staff in the Departments of Oncology and Comparative Medicine for maintaining the SCID mice, and the blood donors and the staff of the Johns Hopkins University Hemapheresis Center. We thank T.C. Wu (Johns Hopkins University, School of Medicine) for his advice and assistance. We acknowledge the support of grants from the National Institute of Allergy and Infectious Diseases (AI45967 to R.A. Cone, HD39613 to J.E.K. Hildreth) and the National Institute of Child Health and Human Development (AI46291 to J.E.K. Hildreth). K.V. Khanna was supported, in part, by a National Research Service Award postdoctoral fellowship from the National Institute on Drug Abuse.

1. Chirgwin, K.D., Feldman, J., Dehovitz, J.A., Minkoff, H., and Landesman, S.H. 1999. Incidence and risk factors for heterosexually acquired HIV in an inner-city cohort of women: temporal association with pregnancy. $J$. Acquir. Immune Defic. Syndr. Hum. Retrovirol. 20:295-299.

2. Sodora, D.L., Gettie, A., Miller, C.J., and Marx, P.A. 1998. Vaginal transmission of SIV: assessing infectivity and hormonal influences in macaques inoculated with cell-free and cell-associated viral stocks. AIDS Res. Hum. Retroviruses. 14(Suppl. 1):S119-S123.

3. Girard, M., et al. 1998. Genital infection of female chimpanzees with human immunodeficiency virus type 1. AIDS Res. Hum. Retroviruses. 14:1357-1367.

4. Tachet, A, et al. 1999. Detection and quantification of HIV-1 in semen: identification of a subpopulation of men at high potential risk of viral sexual transmission. AIDS. 13:823-831.

5. Hart, C.E., et al. 1999. Correlation of human immunodeficiency virus type 1 RNA levels in blood and the female genital tract. J. Infect. Dis. 179:871-882.

6. Nguyen, D.H., and Hildreth, J.E. 2000. Evidence for budding of human 
immunodeficiency virus type 1 selectively from glycolipid-enriched membrane lipid rafts. J. Virol. 74:3264-3272.

7. Krauss, K., and Altevogt, P. 1999. Integrin leukocyte function-associated antigen-1-mediated cell binding can be activated by clustering of membrane rafts. J. Biol. Chem. 274:36921-36927.

8. Manes, S., et al. 1999. Membrane raft domains mediate front-rear polarity in migrating cells. EMBO J. 18:6211-6220.

9. Christian, A.E., et al. 1999. Comparison of the capacity of beta-cyclodextrin derivatives and cyclophanes to shuttle cholesterol between cells and serum lipoproteins. J. Lipid Res. 40:1475-1482.

10. Francis, S.A., et al. 1999. Rapid reduction of MDCK cell cholesterol by methyl-beta-cyclodextrin alters steady state transepithelial electrical resistance. Eur. J. Cell Biol. 78:473-484.

11. Okada, S.S., et al. 1995. Inhibition of human vascular smooth muscle cell migration and proliferation by beta-cyclodextrin tetradecasulfate. J. Pharmacol. Exp. Ther. 273:948-954.

12. Stokes, C.L., Weisz, P.L., Williams, S.K., and Lauffenburger, D.A. 1990 Inhibition of microvascular endothelial cell migration by beta-cyclodextrin tetradecasulfate and hydrocortisone. Microvasc. Res. 40:279-284.

13. Shah, S.M., et al. 1996. Detection of HIV-1 evolution in needles/syringes, paraphenalia, and washes from shooting galleries in Miami: a preliminary laboratory report. J. Acquir. Immune Defic. Syndr. Hum. Retrovirol. 11:301-306.

14. Bosma, G.C., Custer, R.P., and Bosma, M.J. 1983. A severe combined immunodeficiency mutation in the mouse. Nature. 301:527-530.

15. Bosma, M.J., and Carroll, A.M. 1991. The scid mouse mutant: definition, characterization, and potential uses. Annu. Rev. Immunol. 9:323-350.

16. Hyytinen, E., Visakorpi, T., Kallioniemi, A., Kallioniemi, O.P., and Isola, J.J. 1994. Improved technique for analysis of formalin-fixed, paraffin-embedded tumors by fluorescence in situ hybridization. Cytometry. 16:93-99.

17. O'Brien, M.C., and Bolton, W.E. 1995. Comparison of cell viability probes compatible with fixation and permeabilization for combined surface and intracellular staining in flow cytometry. Cytometry. 19:243-255.

18. Mascola, J.R., et al. 2000. Protection of macaques against vaginal transmission of a pathogenic HIV-1/SIV chimeric virus by passive infusion of neutralizing antibodies. Nat. Med. 6:207-210.

19. Marx, P.A., et al. 1996. Progesterone implants enhance SIV vaginal transmission and early virus load. Nat. Med. 2:1084-1089.

20. Howell, A.L., et al. 1997. Human immunodeficiency virus type 1 infection of cells and tissues from the upper and lower human female reproductive tract. J. Virol. 71:3498-3506.

21. Liao, Z., Cimakasky, L.M., Hampton, R., Nguyen, D.H., and Hildreth, J.E.K. 2001. Lipid rafts and HIV pathogenesis: host membrane cholesterol is required for infection by HIV type 1. AIDS Res. Hum. Retroviruses. 17:1009-1019.

22. Tan, X., Pearce-Pratt, R., and Phillips, D.M. 1993. Productive infection of a cervical epithelial cell line with human immunodeficiency virus: implications for sexual transmission. J. Virol. 67:6447-6452.

23. Tan, X., and Phillips, D.M. 1996. Cell-mediated infection of cervix derived epithelial cells with primary isolates of human immunodeficiency virus. Arch. Virol. 141:1177-1189.

24. Bomsel, M. 1997. Transcytosis of infectious human immunodeficiency virus across a tight human epithelial cell line barrier. Nat. Med. 3:42-47.

25. Miller, C.J., and Hu, J. 1999. T cell-tropic simian immunodeficiency virus (SIV) and simian-human immunodeficiency viruses are readily transmitted by vaginal inoculation of rhesus macaques, and Langerhans' cells of the female genital tract are infected with SIV. J. Infect. Dis. 179(Suppl. 3):S413-S417.

26. Hu, J., Gardner, M.B., and Miller, C.J. 2000. Simian immunodeficiency virus rapidly penetrates the cervicovaginal mucosa after intravaginal inoculation and infects intraepithelial dendritic cells. J. Virol. 74:6087-6095.

27. Zhang, Z., et al. 1999. Sexual transmission and propagation of SIV and HIV in resting and activated CD4+ T cells. Science. 286:1353-1357.

28. Anderson, D., and Yunis, E.J. 1983. Trojan horse leukocytes in AIDS N. Engl. J. Med. 309:984-985.

29. Frankel, S.S., et al. 1998. Neutralizing monoclonal antibodies block human immunodeficiency virus type 1 infection of dendritic cells and transmission to T cells. J. Virol. 72:9788-9794.

30. Lehner, T., et al. 1999. The effect of route of immunization on mucosal immunity and protection. J. Infect. Dis. 179(Suppl. 3):S489-S492.

31. Tsai, W.P., Conley, S.R., Kung, H.F., Garrity, R.R., and Nara, P.L. 1996. Preliminary in vitro growth cycle and transmission studies of HIV-1 in an autologous primary cell assay of blood-derived macrophages and peripheral blood mononuclear cells. Virology. 226:205-216.

32. Burkhard, M.J., Obert, L.A., O’Neil, L.L., Diehl, L.J., and Hoover, E.A. 1997. Mucosal transmission of cell-associated and cell-free feline immunodeficiency virus. AIDS Res. Hum. Retroviruses. 13:347-355.

33. Moench, T.R., et al. 1993. The cat/feline immunodeficiency virus model for transmucosal transmission of AIDS: nonoxynol-9 contraceptive jelly blocks transmission by an infected cell inoculum. AIDS. 7:797-802.

34. Markham, R.B., et al. 1996. Selective transmission of human immunodeficiency virus type 1 variants to SCID mice reconstituted with human peripheral blood monoclonal cells. J. Virol. 70:6947-6954.

35. Ibata, B., Parr, E.L., King, N.J., and Parr, M.B. 1997. Migration of foreign lymphocytes from the mouse vagina into the cervicovaginal mucosa and to the iliac lymph nodes. Biol. Reprod. 56:537-543.

36. Zacharopoulos, V.R., Perotti, M.E., and Phillips, D.M. 1997. A role for cell migration in the sexual transmission of HIV-1? Curr. Biol. 7:534-537.

37. Phillips, D.M., Tan, X., Perotti, M.E., and Zacharopoulos, V.R. 1998. Mechanism of monocyte-macrophage-mediated transmission of HIV. AIDS Res. Hum. Retroviruses. 14(Suppl. 1):S67-S70.

38. Toyoda, K., Shoda, T., Uneyama, C., Takada, K., and Takahashi, M. 1997. Carcinogenicity study of beta-cyclodextrin in F344 rats. Food Chem. Toxicol. 35:331-336.

39. Sharma, U.S., Balasubramanian, S.V., and Straubinger, R.M. 1995. Pharmaceutical and physical properties of paclitaxel (Taxol) complexes with cyclodextrins. J. Pharm. Sci. 84:1223-1230. 